Jerome P Gauntlett, Jan B Gutowski, Christopher M Hull et al.

IOP PUBLISHING

Classical AND QuANTUm GRavity

Class. Quantum Grav. 27 (2010) 205018 (15pp)

doi:10.1088/0264-

9381/27/20/205018

\title{
Killing vector fields in three dimensions: a method to solve massive gravity field equations
}

\section{Metin Gurses*}

\author{
Department of Mathematics, Faculty of Sciences, Bilkent University, 06800 Ankara, Turkey \\ E-mail: gurses@fen.bilkent.edu.tr
}

Received 2 February 2010, in final form 20 July 2010

Published 17 September 2010

Online at stacks.iop.org/CQG/27/205018

\begin{abstract}
Killing vector fields in three dimensions play an important role in the construction of the related spacetime geometry. In this work we show that when a three-dimensional geometry admits a Killing vector field then the Ricci tensor of the geometry is determined in terms of the Killing vector field and its scalars. In this way we can generate all products and covariant derivatives at any order of the Ricci tensor. Using this property we give ways to solve the field equations of topologically massive gravity (TMG) and new massive gravity (NMG) introduced recently. In particular when the scalars of the Killing vector field (timelike, spacelike and null cases) are constants then all three-dimensional symmetric tensors of the geometry, the Ricci and Einstein tensors, their covariant derivatives at all orders, and their products of all orders are completely determined by the Killing vector field and the metric. Hence, the corresponding three-dimensional metrics are strong candidates for solving all higher derivative gravitational field equations in three dimensions.
\end{abstract}

PACS numbers: 04.20.Jb, 04.60.Kd, 04.70.Bw

\section{Introduction}

Einstein gravity in three dimensions is trivial because it contains no dynamics. A theory in three dimensions with dynamics is known as topologically massive gravity (TMG) [1, 2]. The action of TMG is the sum of the standard Einstein-Hilbert and Chern-Simons terms in three dimensions. TMG with cosmological constant has been considered recently [3, 4]. This theory violates parity; it is renormalizable but not unitary. Recently a new massive gravity (NMG) theory has been introduced. In the framework of perturbation theory NMG is both renormalizable and unitary [5-7] (see also [8, 9]). Although higher derivative theories play an important role to have a consistent quantum gravity, it is however very difficult to obtain classical solutions of these theories. For this purpose there have been several attempts made

0264-9381/10/205018+15\$30.00 C 2010 IOP Publishing Ltd Printed in the UK \& the USA

to solve these theories, in particular to find black hole solutions. In these attempts different methods were used. In [10] we used perfect fluid solutions of Einstein theory to solve the TMG field equations. In [11-16] by assuming commuting two Killing vector fields Clement gave some solutions of the TMG and NMG field equations. Another method is the principle of symmetric criticality [17-19]. In [20] the algebraic classification of the Ricci tensor is used 
and all known solutions of the TMG are reviewed. In this work the importance of the Killing vector fields in obtaining the solutions of the TMG field equations has also been emphasized. It was shown that (see also [21]) all solutions locally belong to one of three solutions: timelikesquashed $\mathrm{AdS}_{3}$, spacelike-squashed $\mathrm{AdS}_{3}$ and $\mathrm{AdS}$ pp-waves [22-24]. In [25] Chow et al used the Kundt metrics to solve TMG field equations and they obtained new solutions of TMG. Another method is to use the Godel-type metrics [" 26-28] in three dimensions [29]. There are also some other attempts [30-32] made to solve the field equations of TMG. AdS pp-wave solutions of the NMG field equations were considered in [33]. The importance of the Killing vectors in finding the solutions of the massive gravity field equations in three dimensions is also observed in some of these works. If they exist, Killing vectors in three dimensions are not only important in the derivation of the exact solutions but they also control the whole geometry of spacetime.

In this work we shall introduce a new method for three-dimensional gravity theories. The starting point is to assume a one parameter family of isometry. This implies the existence of a Killing vector field in the spacetime geometry. In general this assumption may simplify and reduce the number of the field equations by choosing a suitable coordinate patch so that one coordinate in the line element becomes cyclic. In the case of four- and higher-dimensional geometries this may give information about some components of the curvature and hence the Ricci tensors, but it does not provide all components of these tensors. We shall show that this is possible in three dimensions.

If a three-dimensional spacetime admits a non-null Killing vector field, writing it in terms of two scalars, its norm and rotation scalar, we show that the metric and the Ricci tensors are completely determined in terms of the Killing vector, its scalars and vectors obtained through these scalars (theorem 1). Physical interpretation of the corresponding spacetime metric is that it solves the Einstein-Maxwell-dilaton field equations in three dimensions. When the scalars of the Killing vector field become constant then the corresponding Ricci tensor becomes relatively simple (theorem 2 ). We show that the spacetime metric, in this case, solves the field equations of TMG and NMG. Indeed these metrics (for timelike and spacelike cases) not only solve these field equations, but they also solve all higher (curvature) derivative theories at any order. When the Killing vector field is null we have a similar result (theorem 3). Without referring to the metric tensor we construct the Einstein tensor when the spacetime geometry admits a null vector. The corresponding metric solves Einstein field equations with a null fluid distribution and an exotic scalar field. When the rotation scalar of the null Killing vector field becomes constant the Einstein tensor becomes simpler (theorem 4) and the metric solves the field equations of the TMG and NMG. Both for non-null and null cases when the scalars of the Killing vector fields become constant then the metric solves not only the field equations of the TMG and NMG but also all higher derivative gravitational field equations in three dimensions.

In section 2 we give Ricci and metric tensors in terms of the non-null Killing vector field and its scalars. The Einstein tensor represents a charged fluid with a dilaton field. In particular, if the scalars are constants the Ricci tensor is expressed in terms of the Killing vector field and the Gaussian curvature of the two-dimensional background space in section 3. In section 4 we show that when the Killing vector field is null then the Ricci tensor is calculated in terms of the null Killing vector field and its rotation scalar. In section 5 we investigate the 
case when the rotation scalar is a constant. In sections 6 and 7, by using Ricci tensors found in the previous sections, we solve the field equations of the TMG and NMG respectively.

\section{Non-null Killing vectors in three dimensions}

In this section we shall construct the metric and Einstein tensors in terms of scalar functions of a non-null Killing vector. Our conventions in this paper are similar to the convention of Hawking-Ellis [34].

Let $\mathrm{u}^{\mu}$ be a non-null Killing vector field satisfying the Killing equation

$$
\mathrm{u}_{\mu, v}+\mathrm{u}_{v} ; \mu=0
$$

with $u_{\alpha} u^{\alpha}=\alpha \neq 0$. One can show that

$$
u^{\alpha} u_{\alpha ; \mu}=\frac{1}{2} \alpha_{, \mu}, \quad u^{\alpha} u_{\mu ; \alpha}=-\frac{1}{2} \alpha_{, \mu} .
$$

The norm function $\alpha$ is in general not a constant. It is possible to write the Killing equation as

$$
u_{\mu ; \nu}=\frac{1}{2}\left(u_{\mu ; v}-u_{v ; \mu}\right)=\eta_{\mu \nu \alpha} v^{\alpha}
$$

where $v^{\alpha}$ is any vector field and $\eta_{\mu v \alpha}$ is the Levi-Civita alternating symbol. Using (2) we find that

$$
\mathrm{v} \rho=\mathrm{wu}_{\rho}+\eta_{\rho \mu \sigma} \varphi, \mu \mathbf{u}_{\sigma},
$$

where $^{w}=\frac{u_{\mu} v^{\mu}}{\alpha}$. Using (4) in (3) we get

$$
\mathrm{u}_{\mu ; \nu}=\mathrm{W} \eta_{\mu v \alpha} \mathrm{u} \alpha+\mathrm{u}_{\mu} \varphi, v-\mathrm{u} v \varphi, \mu,
$$

where $\phi=\frac{1}{2} \ln \alpha$. The functions $w$ and $\varphi$ specify the Killing vector field and they are assumed to be linearly independent. Differentiating (5) with respect to $\mathrm{x}^{\sigma}$ and anti-symmetrizing both sides with respect to $v$ and $\sigma$ we get

$\mathrm{u}_{\mu ; v \sigma}-\mathrm{u}_{\mu ; \sigma v}=\mathrm{w}, \sigma \eta_{\mu v \alpha} \mathrm{u}_{\alpha}-\mathrm{w}, v \eta_{\mu \sigma \alpha} \mathrm{u} \alpha-\mathrm{w} \eta_{\mu v \alpha} \varphi_{\alpha} \mathrm{u}, \sigma+\mathrm{w} \eta_{\mu \sigma \alpha} \varphi_{\alpha} \mathrm{u}_{v}-2 \eta_{v \sigma \alpha} \mathrm{u} \alpha \varphi, \mu$

$$
-\mathrm{u}_{v} \varphi, \sigma \varphi, \mu+\mathrm{u} \sigma \varphi, \mu \varphi, v-\mathrm{u} v \varphi ; \mu \sigma+\mathrm{u} \sigma \varphi ; \mu v+\mathrm{W} 2\left(\mathrm{~g}_{\mu \nu} \mathrm{u} \sigma-\mathrm{g}_{\mu \sigma} \mathrm{u} v\right)
$$

Using the identities

$$
\begin{aligned}
& \mathrm{W}, \sigma \eta_{\mu v \alpha} \mathrm{u} \alpha+\mathrm{W}, \mu \eta_{v \sigma \alpha} \mathrm{u} \alpha+\mathrm{W}, v \eta_{\sigma \mu \alpha} \mathrm{u}_{\alpha}=\eta_{\sigma \mu \nu} \mathrm{u} \alpha \varphi_{, \alpha}=0, \eta_{\mu \nu \alpha} \varphi_{\alpha} \mathrm{u}, \sigma+ \\
& \eta_{\nu \sigma \alpha} \varphi \alpha \mathrm{u}, \mu+\eta_{\sigma \mu \alpha} \varphi \alpha \mathrm{u}, v=\eta_{\sigma \mu \nu} \varphi_{\alpha} \mathrm{u} \alpha=0
\end{aligned}
$$

equation (6) reduces to (here $\varphi^{\alpha}=\mathrm{g}^{\alpha \mu} \varphi_{, \mu}$ )

$$
\begin{aligned}
& \mathrm{u}_{\mu ; \nu} \quad-\quad \mathrm{\sigma u}_{\mu ; \sigma v}=\mathrm{w} \eta_{\nu \sigma \alpha} \varphi \mathrm{u}_{\mu}-\eta_{\nu \sigma \alpha} \mathrm{u} \mathrm{y}_{\mu}+\varphi, \mu\left(\mathrm{u}_{\sigma} \varphi, v-\mathrm{u}_{v} \varphi, \sigma\right) \\
& \quad+\mathrm{w}\left(\mathrm{g}_{\mu \nu} \mathrm{u}_{\sigma}-\mathrm{g}_{\mu \sigma} \mathrm{u} v\right),
\end{aligned}
$$

where 


$$
\mathrm{y}_{\mu}=\mathrm{w}, \mu+2 \mathrm{w} \varphi, \mu .
$$

Using the Ricci identity $\mathrm{u}_{\mu ; \nu \sigma}-\mathrm{u}_{\mu ; \sigma v}=\mathrm{R}_{\mu \nu \sigma}^{\rho} \mathrm{u}_{\rho}$ in (9) we get

$\mathrm{R}_{\rho} \mu \nu \sigma \mathrm{u}_{\rho}=\mathrm{w} \eta_{\nu \sigma \alpha} \varphi \alpha \mathrm{u}_{\mu}-\eta_{\nu \sigma \alpha} \mathrm{u} \alpha \mathrm{y} \mu+\varphi, \mu(\mathrm{u} \sigma \varphi, v-\mathrm{u} v \varphi, \sigma)+\mathrm{W} 2\left(\mathrm{~g}_{\mu \nu} \mathrm{u} \sigma-\mathrm{g}_{\mu \sigma} \mathrm{u} v\right)$

and hence we obtain

$$
\mathrm{R}^{\rho}{ }_{v} \mathrm{u}_{\rho}=-\zeta_{v}-\left(\nabla^{2} \varphi+2 \mathrm{w}^{2}\right) \mathrm{u}_{v}
$$

where

$$
\zeta_{\mu}=\eta_{\mu \alpha \beta} \text { ya } \mathbf{u} \beta .
$$

In three dimensions the Weyl tensor vanishes and hence the curvature tensor is expressed solely in terms of the Ricci tensor

$R_{\rho \mu \nu \alpha}=-R_{\mu \nu} g_{\rho \alpha}+R_{\rho \nu} g_{\mu \alpha}+R_{\mu \alpha} g_{\rho v}-R_{\rho \alpha} g_{\mu \nu}+\frac{R}{2}\left(g_{\rho \alpha} g_{\mu \nu}-g_{\rho v} g_{\mu \alpha}\right)$.

Combining equations (11) and (14) we obtain

$$
\bar{R}_{\mu \nu} u_{\sigma}-\bar{R}_{\mu \sigma} u_{v}+\zeta_{\sigma} g_{\mu \nu}-\zeta_{\nu} g_{\mu \sigma}=-\eta_{\nu \sigma \alpha} u^{\alpha} y_{\mu}+w \eta_{\nu \sigma \alpha} \phi^{\alpha} u_{\mu}
$$

where

$$
\begin{aligned}
& \bar{R}_{\mu \nu}=-R_{\mu \nu}+\left(P+\frac{R}{2}-w^{2}\right) g_{\mu \nu}-\phi_{; \mu \nu}-\phi_{, \mu} \phi_{, \nu}, \\
& P=\nabla^{2} \phi+2 \phi_{, \alpha} \phi^{\alpha}+2 w^{2} .
\end{aligned}
$$

(17)

In addition to identities (7) we also have the following identities:

$$
\begin{aligned}
& \mathrm{Z} \mu \mathrm{U} \sigma-\mathrm{Z} \sigma \mathrm{U} v=\alpha \eta_{\mu \sigma \rho} \varphi_{\rho}, \zeta_{\mu} \mathrm{y}_{\sigma}-\zeta_{\sigma} \\
& \mathrm{y} v=-(\mathrm{y} \alpha \mathrm{y} \alpha) \eta_{\mu \sigma \rho} \mathrm{u}_{\rho} .
\end{aligned}
$$

Using these identities in (15) we arrive at the following theorem.

Theorem 1. If a three-dimensional spacetime admits a non-null Killing vector field $\mathrm{u}^{\mu}$, then its metric and the Einstein tensors are respectively given by

$g_{\mu \nu}=\frac{1}{\alpha} u_{\mu} u_{v}+\frac{1}{\zeta^{\alpha} \zeta_{\alpha}} \zeta_{\mu} \zeta_{\nu}+\frac{1}{y_{\alpha} y^{\alpha}} y_{\mu} y_{v}$

$G_{\mu \nu}=-\frac{H}{\alpha} u_{\mu} u_{v}+\frac{1}{\alpha}\left[\left(w z_{\mu}-\zeta_{\mu}\right) u_{v}+\left(w z_{v}-\zeta_{\mu}\right) u_{\mu}\right.$

$$
+\left(\nabla^{2} \phi+w^{2}\right) g_{\mu \nu}-\phi_{, \mu} \phi_{, v}-\phi_{; \mu \nu},
$$

$H=2 \nabla^{2} \phi+3 w^{2}+\frac{1}{2} R-\phi^{\alpha} \phi_{, \alpha}$,

$z_{\mu}=\eta_{\mu \alpha \beta} \phi^{\alpha} u^{\beta}$,

where $R$ is the Ricci scalar provided that the functions $\mathrm{w}$ and $\varphi$ are not constant at the same time. Here we assume that $\alpha$ nowhere vanishes in the spacetime. 
Remark 1. When $\mathrm{u}^{\mu}$ is a timelike Killing vector field, letting $\alpha=-\mathrm{e}^{2 \varphi}$, then it can be shown that the source of the Einstein equations is composed of a charged fluid distribution and a dilaton field $\varphi$ (in the Einstein frame). The electromagnetic field tensor $f_{\mu v}$ is given by $f_{\mu v}=w$ $\eta_{\mu v \alpha} \mathrm{u} \alpha-\mathrm{u}_{\mu} \varphi, v+\mathrm{u} v \varphi, \mu$

so that

$$
\mathrm{f}^{\mu v} ; \mu=-\zeta^{v}+\left(\nabla^{2} \varphi-2 \mathrm{w}^{2}\right) \mathrm{u}^{v}
$$

The Maxwell energy momentum tensor is given as $M_{\mu v}=-\frac{1}{2} \alpha\left(w^{2}+\phi_{, \alpha} \phi^{\alpha}\right) g_{\mu v}+\left(w^{2}+\phi_{, \alpha} \phi^{\alpha}\right) u_{\mu} u_{v}+\alpha \phi_{, \mu} \phi_{, v}-w\left(z_{\mu} u_{v}+z_{v} u_{\mu}\right)$.

Then the field equations are

$$
\begin{aligned}
& G_{\mu \nu}=\frac{1}{4} \mathrm{e}^{-2 \phi} M_{\mu \nu}+\mathrm{e}^{-2 \phi}\left(-2 \nabla^{2} \phi+4 \phi, \alpha\right. \\
&\left.\phi^{\alpha}+\mathrm{e}^{-2 \phi} f^{2}-\frac{R}{2}\right) u_{\mu} u_{v} \\
&+\left(\nabla^{2} \phi+\frac{1}{4} \mathrm{e}^{-2 \phi} f^{2}\right) g_{\mu \nu}-\mathrm{e}^{-2 \phi}\left(J_{\mu} u_{v}+J_{v} u_{\mu}\right)-\phi_{; \mu \nu},
\end{aligned}
$$

where $\mathrm{J}^{\mu}=\mathrm{f}^{\alpha \mu} ; \alpha$ and $\mathrm{f}^{2}=\mathrm{f}_{\alpha \beta} \mathrm{f}^{\alpha \beta}$.

All our analysis so far is valid when the norm $\alpha$ of the Killing vector field $\mathrm{u}^{\mu}$ is different than zero everywhere in spacetime. If there are points where $\alpha=0$ we exclude them from the spacetime manifold for causality reasons. This set of points defines the Killing horizons which are in general coordinate singularities of the spacetime [34]. We may find suitable coordinate patches where these coordinate singularities are removed (maximal extension) [35]. Examples of such Killing horizons in three dimensions are discussed in the BTZ spacetime [36]. The Killing horizon in general is a subset of the set of fixed points of the isometry whose generator is the Killing vector $u$. Such a set is called the local isometry horizon (LIH) by Carter [35]. The fixed points of an isometry are the set of points where the Killing vector field vanishes, i.e. critical points of $u, \mathrm{u}(\mathrm{p})=0$. It is obvious that our analysis fails at the points of the LIH.

For a given Killing vector field we have [37]

$$
\mathrm{u}_{\mu, \alpha \beta}=\mathrm{u}_{\rho} \mathrm{R}_{\beta \mu \alpha}^{\rho} .
$$

At the critical points of $u$ the second covariant derivative of the Killing vector field vanishes. This implies that all higher derivatives of $u$ at $p$ are either zero or expressed in terms of the first partial derivative $\mathrm{F}_{\mu v}(\mathrm{p})=\mathrm{u}_{\mu, v}(\mathrm{p})$. The integrability of (29) at $p$ gives

$$
\mathrm{F}_{\mu \alpha}(\mathrm{p}) \mathrm{R}^{\alpha}{ }_{v}+\mathrm{F}_{v \alpha}(\mathrm{p}) \mathrm{R}^{\alpha}{ }_{\mu}=0
$$

which imply that the Segre type of the Ricci tensor is special at the critical points of $u$ [38, 39]. In the neighborhood $U$ of the critical point $p$ the Killing vector has the linearized form

$$
\mathrm{u}_{\mu}=\mathrm{F}_{\mu \alpha}(\mathrm{p}) \mathrm{Z}^{\alpha},
$$

where $Z^{\mu}=x^{\mu}-p^{\mu}$. Theorem 1 is valid in $U$ with

$$
\alpha=F_{\alpha}^{\mu}(p) F_{\mu \beta}(p) Z^{\alpha} Z^{\beta}, \quad w=-\frac{1}{2 \alpha} \eta^{\mu v \alpha} F_{\mu v}(p) F_{\alpha \beta}(p) Z^{\beta} .
$$


To identify the real structure of the spacetime about the critical points for a given particular exact solution it may be useful to study the maximal extension of the corresponding coordinate patches.

If the norm $\alpha$ of a Killing vector field is constant everywhere in spacetime, then there are no fixed points of the corresponding isometry. Then theorem 1 is valid everywhere in spacetime. This is the case which will be considered in the next sections. This will be true not only for spacelike and timelike Killing vector fields but also for null Killing vector fields.

\section{Non-null case with constant scalars}

In the previous section we assumed that the scalars $w$ and $\varphi$ of the Killing vector field are not simultaneously constant. If $\mathrm{w}$ and $\varphi$ are both constants, then we obtain

$$
\mathrm{u}_{\mu ; \nu}=\mathrm{W} \eta_{\mu \nu \alpha} \mathbf{u} \alpha .
$$

Then following the above procedure and using (21) we have the following result.

Theorem 2. If a three-dimensional spacetime admits a non-null Killing vector field $\mathrm{u}^{\mu}$ with both $\mathrm{w}$ and $\alpha$ being constants, then the Einstein tensor is given as

$$
G_{\mu \nu}=-\frac{1}{\alpha}\left(3 w^{2}+\frac{1}{2} R\right) u_{\mu} u_{\nu}+w^{2} g_{\mu \nu} \text {. }
$$

In this case, since the vector $\zeta_{\mu}$ vanishes in (15), the metric tensor is not obtained.

Remark 2. If $u^{\mu}$ is a timelike Killing vector field the above Einstein tensor represents a dust distribution in a spacetime with a positive cosmological constant.

For completeness let us give the spacetime metrics of each case: we shall consider the case where $\alpha$ and $\mathrm{w}$ are the constants.

1. Let $u^{u^{\mu}}=-\frac{1}{u_{0}} \delta_{0}^{\mu}$ be the timelike vector field then $u^{\mu} u_{\mu}=-1$. Hence, $\alpha=-1$ and $u_{\mu}=g_{\mu \alpha}$ $\mathrm{u}^{\alpha}$; then $g_{00}=-u_{0}^{2}, g_{01}=-u_{0} u_{1}, g_{02}=-u_{0} u_{2}$. Then the spacetime metric can be taken as $\left(\mathrm{x}^{\mu}=(\mathrm{t}, \mathrm{r}, \mathrm{z})\right)$ :

$$
d s^{2}=-\left(u_{0} d t+u_{1} d r+u_{2} d z\right)^{2}+M^{2} d r^{2}+2 L d r d z+N^{2} d z^{2}
$$

where $M^{2}=g_{11}+u_{1}^{2}, N^{2}=g_{22}+u_{2}^{2}, L=g_{12}+u_{1} u_{2}$. Here $u_{0}$ is a constant (due to the Killing equation) and the metric functions $M, N, L, u_{1}$ and $u_{2}$ depend on the variables $r$ and $z$. The metric in (35) is of the Godel type which was used in [“29]. The Einstein tensor is given in (34) with $\alpha=-1$. The only field equation is

$$
u_{2, r}=u_{1, z}+2 w \Delta / u_{0}
$$

where $\Delta=\left|u_{0}\right| \sqrt{ } M^{2} N^{2}-L^{2}$.For simplicity consider the

case $\mathrm{L}=0$. Then

$\mathrm{R}=2 \mathrm{~K}+2 \mathrm{w}^{2}$ where KistheGaussiancurvatureofthelocallyEuclidiantwo-dimensional background space with the line element $\mathrm{d}_{2}^{2}=M^{2} \mathrm{~d} r^{2}+N^{2} \mathrm{~d} z^{2}$. Metric (35) gives the 
Einstein tensor (34) where the only field equation is given in equation (36) with $\alpha=-1$ and $\mathrm{w}$ is constant.

2. Let $u^{\mu}=\frac{1}{u_{2}} \delta_{2}^{\mu}$ be the spacelike vector field; then $u^{\mu} u_{\mu}=1$. Hence $\alpha=1$ and $u_{\mu}=g_{\mu \alpha} u^{\alpha}=\frac{1}{u_{2}} g_{\mu 2}$; then $g_{22}=u_{2}^{2}, g_{12}=u_{1} u_{2}, g_{02}=u_{0} u_{2}$. Then the spacetime metric can be taken as

$$
d s^{2}=\left(u_{2} d z+u_{1} d r+u_{0} d t\right)^{2}+M^{2} d r^{2}+2 L d r d t-N^{2} d t^{2},
$$

where $M^{2}=g_{11}-u_{1}^{2}, N^{2}=u_{0}^{2}-g_{00}, L=g_{01}-u_{0} u_{1}, u_{2}$ is a constant (due to the Killing equation) and the metric functions $M, N, L, u_{1}$ and $u_{0}$ depend on the variables $t$ and $r$. The Einstein tensor is given in (34) with $\alpha=1$. The only field equation is

$$
u_{0, r}=u_{1, t}+2 w \Delta / u_{2}
$$

where $\quad-\quad \Delta=\left|u_{2}\right| \sqrt{ } M^{2} N^{2}+L^{2}$. In both cases the Ricci scalar $R$ is not constant. In this case as well take $\mathrm{L}=0$ for simplicity then we have $\mathrm{R}=2 \mathrm{~K}+$ $2 \mathrm{w}^{2}$ where $K$ is the Gaussian curvature of two-dimensional locally Minkowskian background space with the line element $\mathrm{d} s_{2}^{2}=M^{2} \mathrm{~d} r^{2}-N^{2} \mathrm{~d} t^{2}$. Metric (37) gives the Einstein tensor (34) where the only field equation is given in (38) with $\alpha=1$ and w being the constant. In both cases the Ricci and Einstein tensors are respectively given as

$$
\begin{aligned}
& R_{\mu \nu}=-\frac{1}{\alpha}\left(4 w^{2}+K\right) u_{\mu} u_{v}+\left(2 w^{2}+K\right) g_{\mu \nu} \\
& G_{\mu \nu}=-\frac{1}{\alpha}\left(4 w^{2}+K\right) u_{\mu} u_{v}+w^{2} g_{\mu \nu} .
\end{aligned}
$$

Itispossibletopassfromthetimelikecasetothespacelikecasebycomplextransformation [13]. When the background two-dimensional spaces are de Sitter or anti-de Sitter, in each case, these solutions are known as timelike squashed $\mathrm{AdS}_{3}$ and spacelike squashed $\mathrm{AdS}_{3}$, respectively [11, 20, 21].

When $R$ is a constant, the Cotton tensor and the Laplacian of the Ricci tensor take simple forms (for both cases). They will be used later:

$$
\begin{aligned}
& C_{\mu v}=-w\left(4 w^{2}+K\right)\left[g_{\mu v}-\frac{3}{\alpha} u_{\mu} u_{v}\right], \\
& \nabla^{2} R_{\mu \nu}=-2 w^{2}\left(4 w^{2}+K\right)\left[g_{\mu \nu}-\frac{3}{\alpha} u_{\mu} u_{v}\right]
\end{aligned}
$$

\section{Three-dimensional spacetime admitting a null Killing vector field}

In this section we shall construct the Einstein tensor with respect to the scalar functions of a null Killing vector field.

Let $\xi_{\mu}$ be the Killing vector field. It satisfies the Killing equation 


$$
\xi_{\mu ; \nu}=\frac{1}{2}\left(\xi_{\mu ; \nu} \quad \xi_{v ; \mu}\right)=\eta_{\mu \nu \alpha} v^{\alpha}
$$

where $\eta_{\mu \nu \alpha}=\sqrt{ }-\operatorname{det}(g) \epsilon_{\mu \nu \alpha}$ and $\mathrm{v}^{\mu}$ is an arbitrary vector field and a semicolon defines the covariant derivative with respect to the metric $\mathrm{g}_{\mu v}$. Here $\epsilon_{\mu \nu \alpha}$ is the three-dimensional LeviCivita alternating symbol. Since $\xi^{\alpha} \xi_{\alpha ; \beta}=0$ and $\xi^{\alpha} \xi_{\beta ; \alpha}=0$, then $\mathrm{v}^{\mu}=w \xi^{\mu}$. Hence

(43) becomes

$$
\xi_{\mu ; \nu}=\mathrm{w} \eta_{\mu \nu \alpha} \xi_{\alpha},
$$

where $w$ is an arbitrary function. Taking one more covariant derivative of (44) we find that

$$
\xi_{\mu ; \nu \alpha}=\mathrm{w}, \alpha \eta_{\mu \nu \rho} \xi_{\rho}-\mathrm{w} 2\left(\mathrm{~g}_{\mu \alpha} \xi_{\nu}-\mathrm{g}_{\nu \alpha} \xi_{\mu}\right) .
$$

Using this equation and the Ricci identity we get

$\xi_{\mu, v \alpha}-\xi_{\mu ; \alpha \nu}=\mathrm{w}, \alpha \eta_{\mu \nu \rho} \xi_{\rho}-\mathrm{w}, v \eta_{\mu \alpha \rho} \xi_{\rho}-\mathrm{w}_{2}\left(\mathrm{~g}_{\mu \alpha} \xi_{\nu}-\mathrm{g}_{\nu \mu} \xi_{\alpha}\right)=\mathrm{R}_{\rho} \mu \nu \alpha \xi_{\rho}$.

On the other hand, since the Weyl tensor vanishes in three dimensions the Riemann tensor is expressed totally in terms of the Ricci tensor as expressed in (14). Using (46) and (14) we find

$$
\mathrm{Q}_{\mu \nu} \xi_{\alpha}-\mathrm{Q}_{\mu \alpha} \xi_{\nu}-\zeta_{\nu} \mathrm{g}_{\mu \alpha}+\zeta_{\alpha} \mathrm{g}_{\mu \nu}=\mathrm{W}, \alpha \eta_{\mu \nu \rho} \xi_{\rho}-\mathrm{w}, v \eta_{\mu \alpha \rho} \xi_{\rho},
$$

where

$$
\begin{aligned}
\zeta_{\mu} & =\eta_{\mu \alpha \beta} \mathrm{w} \alpha_{\alpha} \xi_{\beta}, \\
Q_{\mu \nu} & =-R_{\mu \nu}+\left(w^{2}+\frac{R}{2}\right) g_{\mu \nu} .
\end{aligned}
$$

Here $R$ is the Ricci scalar and $\mathrm{w}^{\alpha}=\mathrm{g}^{\alpha \mu} \mathrm{w}_{, \mu}$. Let the vector $X_{\mu}$ be defined as

$$
\mathrm{X}_{\mu}=\eta_{\mu \alpha \beta} \mathrm{F}_{\alpha} \xi_{\beta},
$$

where $\mathrm{F}^{\mu}=\mathrm{g}^{\mu \alpha} \mathrm{F}_{, \alpha}$ and $F$ is any function independent of $t$. It is easy to show that $\xi^{\mu} \mathrm{X}_{\mu}=\mathrm{X}^{\mu} \mathrm{X}_{\mu}$ $=0$. Since the orthogonal null vectors can only be parallel, then we have

$$
X_{\mu}=\sigma \xi_{\mu} .
$$

Hence,

$$
\zeta_{\mu}=\psi \xi_{\mu}
$$

and

$$
\xi_{\mu} w_{, v}-\xi_{v} w_{, \mu}=\psi \eta_{\mu \nu \alpha} \xi^{\alpha}, \quad \psi \neq 0
$$

which also leads to

$$
\mathrm{W} \alpha \mathrm{W}, \alpha=\psi 2 .
$$

Using (53) in (47) we obtain 


$$
Q_{\mu \nu}+\psi g_{\mu \nu}-\frac{1}{\psi} w_{, u} w_{, v}+\rho \xi_{\mu} \xi_{v}=0
$$

Hence,

$$
R_{\mu \nu}=\rho \xi_{\mu} \xi_{v}-\left(\psi+2 w^{2}\right) g_{\mu v}-\frac{1}{\psi} w_{, u} w_{, v},
$$

where $\rho$ is a function to be determined and the Ricci scalar is given by

$$
R=-6 w^{2}-4 \psi
$$

Then we have the following theorem.

Theorem 3. Let $\xi^{\mu}$ be a null Killing vector field of a three-dimensional spacetime; then the corresponding Einstein tensor is given by

$$
G_{\mu \nu}=\rho \xi_{\mu} \xi_{v}-\frac{1}{\psi} w_{, u} w_{, v}+\left(w^{2}+\psi\right) g_{\mu \nu},
$$

where $\rho$ is a function to be determined. Furthermore the scalar field satisfies the following partial differential equation:

$$
\nabla_{\mu}\left(\frac{1}{\psi} w^{\mu}\right)=2 w
$$

It is quite interesting that by imposing a null Killing vector in a three-dimensional spacetime geometryweobtaintheformoftheEinsteintensorwithoutknowingthemetrictensorexplicitly, except the function $\rho$. The source of the Einstein field equations is composed of a null fluid and a scalar field. The scalar field satisfies a nonlinear differential equation (59). From the Einstein equations the energy momentum tensor $\mathrm{T}_{\mu \nu}$ of the source satisfies $\mathrm{T}_{\mu \nu} \xi^{\mu} \xi^{v}=0$. For any timelike unit vector field $v u^{\mu}$ we have $T_{\mu v} u^{\mu} u^{v}=\rho\left(\xi^{\mu} u_{\mu}\right)^{2}-\psi^{1}\left(w_{, \mu} u^{\mu}\right)^{2}+\left(\psi+w^{2}\right)$.

Choosing $\psi=-\mathrm{w}_{, \mu} \mathrm{W}^{\mu}$ the sign of the energy depends on the sign of the function $\rho$.

As a summary we can conclude this section as: if $\xi_{\mu}$ is a null Killing vector field of a three-dimensional spacetime geometry, then the corresponding metric solves the Einstein null fluid scalar field equations. The only field equations are (44) and the scalar field equation given in equation (59).

\section{Null case without the scalar field}

In the previous section we considered the case $\psi \neq 0$. Vanishing of $\psi$ is a distinct case. Due to this reason we consider it in a separate section. We have the following result:

Theorem 4. When the scalar function $\psi$ in theorem 3 is zero then $\mathrm{w}$ becomes a constant and the Ricci and Einstein tensors take the simple forms

$$
\begin{aligned}
R_{\mu v} & =\rho \xi_{\mu} \xi_{v}-2 w^{2} g_{\mu v} \\
\text { (60) } \quad G_{\mu v} & =\rho \xi_{\mu} \xi_{v}+w^{2} g_{\mu v},
\end{aligned}
$$

with $\mathrm{R}=-6 \mathrm{w}^{2}$.

For explicit solutions we shall adopt some simplifying assumptions. 
(5.A). As an example let us assume that $\xi^{\mu}=\delta_{0}^{\mu}$. Then $\xi_{\mu}=\mathrm{g}_{\mu \alpha} \xi^{\alpha}=\mathrm{g}_{\mu 0}$. Since $\xi_{\mu}$ is a null vector, $\xi_{0}=0$. Hence, we get $\mathrm{g}_{00}=0, \mathrm{~g}_{01}=\xi_{1}, \mathrm{~g}_{02}=\xi_{2}$. The corresponding spacetime metric can be given as $\left(\mathrm{x}^{\mu}=(\mathrm{t}, \mathrm{r}, \mathrm{z})\right)$

$$
\mathrm{d}^{2}=2\left(\xi_{\mu} \mathrm{d} x^{\mu}\right) \mathrm{d} t+m^{2} \mathrm{~d} r^{2}+n^{2} \mathrm{~d} z^{2}+2 \ell \mathrm{d} r \mathrm{~d} z
$$

where $\xi_{1}, \xi_{2}, m, n$ and are the functions of $r$ and z. Let $\quad \ell=0$; then det $\equiv-\Delta^{2}=(\mathrm{g})$ $-\left(\xi_{1}^{2} n^{2}+\xi_{2}^{2} m^{2}\right)$, and the function $\psi$ defined in (52) takes the form $\psi=\frac{\xi_{1} w_{2}-\xi_{2} w_{r},}{\Delta}$ and $g_{\mu \nu}=\left(\begin{array}{lll}0 & \xi_{1} & \xi_{2} \\ \xi_{1} & m^{2} & 0 \\ \xi_{2} & 0 & n^{2}\end{array}\right), \quad g^{\mu \nu}=\frac{1}{\Delta^{2}}\left(\begin{array}{lll}-m^{2} n^{2} & \xi_{1} n^{2} & \xi_{2} m^{2} \\ \xi_{1} n^{2} & \xi_{2}^{2} & -\xi_{1} \xi_{2} \\ \xi_{2} m^{2} & -\xi_{1} \xi_{2} & \xi_{1}^{2}\end{array}\right)$.

Equation (44) reduces to

$$
\xi_{1, z}-\xi_{2, r}=2 w \Delta \text {. }
$$

The function $\rho$ can be calculated in terms of the metric functions $\xi_{1}, \xi_{2}, \mathrm{~m}$ and $n$. By using a transformation $r=f(R, Z)$ and $z=g(R, Z)$ where $f$ and $g$ are any differentiable functions, without losing any generality, we can let one of the functions $\xi_{1}$ or $\xi_{2}$ be zero. Here in this work we will take $\xi_{1}=0$ and let $\xi_{2}=\mathrm{q}$. With these simplifications we obtain $\Delta=q m_{\mathrm{q}_{\mathrm{r}} \mathrm{r}}=$ $-2 \mathrm{w} \mathrm{q} \mathrm{m}$, and

$$
\mathrm{ds}^{2}=2 \mathrm{qdzdt}+\mathrm{m}^{2} \mathrm{dr}^{2}+\mathrm{n}^{2} \mathrm{dz}^{2} .
$$

where $\mathrm{w}$ is a nonzero constant. With such a choice we have $\rho=\frac{1}{m^{3} q^{3}}\left[2 w q m^{2} n n_{, r}+n q m_{, r} n_{, r}-m^{2} q m_{, z z}+m^{2} m_{, z} q_{, z}-m n q n_{, r r}-m q\left(n_{, r}\right)^{2}\right]$.

Remark 3. If $\mathrm{w}=0$, the Killing vector becomes hypersurface orthogonal and $q$ becomes a constant. Then

$$
\rho=\frac{1}{m^{3} q^{2}}\left[n q m_{, r} n_{, r}-m^{2} m_{, z z}+m^{2} m_{, z} q_{, z}-m n n_{, r r}-m\left(n_{, r}\right)^{2}\right] .
$$

(5.B). Letting $\mathrm{q}=\mathrm{e}^{\mathrm{y}}$ and $\mathrm{n}=\mathrm{m}$ we get $m=-\frac{1}{2 w} y_{r}, w \neq 0$. Here $y$ is a function of $r$ and $\mathrm{z}$. Then

$$
\rho=\frac{1}{\mathrm{e}^{2 y} y_{, r}}\left[-\left(y_{, z z}+y_{, r r}\right)+\frac{1}{2}\left(\left(y_{, r}\right)^{2}+\left(y_{, z}\right)^{2}\right)\right]_{, r} .
$$

We get a nonlinear partial differential equation for $y$ when $\rho=\rho_{0}$ is a constant:

$$
-\left(y_{, z z}+y_{, r r}\right)+\frac{1}{2}\left(\left(y_{, r}\right)^{2}+\left(y_{, z}\right)^{2}\right)=\frac{1}{2} \rho_{0} \mathrm{e}^{2 y}+\rho_{1},
$$


where $\rho_{1}$ is an integration constant. An exact solution of the above equation is given as $y=$ $-\ln \left[\mathrm{A}+\mathrm{B}\left(\mathrm{r} \rightarrow-\mathrm{V}_{\underline{0}}\right)^{2}\right]$ where $\rightarrow v_{\rho_{1}}=0$ and $\rho_{0}==4 \mathrm{AB}$. After performing some scale $=$ transformations z Az,r Ar and letting B w and $\mathrm{r}_{0} 0$ the metric becomes

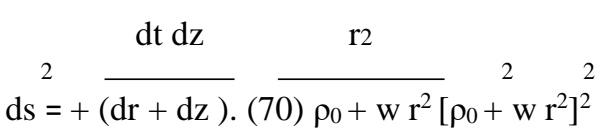

This metric solves Einstein field equations (61) with null fluid whose energy density $\rho=\rho_{0}$ is a constant. All curvature invariants are constants. For instance $R=-6 w^{2}, R^{\alpha \beta} R_{\alpha \beta}=12 w^{4}$. Hence, the spacetime is not asymptotically flat. When $\rho_{0}=0$ the solution corresponds to $\operatorname{AdS}_{3}$. (5.C). When $w=0$, the Killing vector becomes covariantly constant. By taking $m=n$ the function $\rho$ becomes

$$
\rho=-\frac{1}{m q}\left(m_{z z}+m_{r r}\right)
$$

and the metric takes the form

$$
d s^{2}=2 q d z d t+m^{2}\left(d r^{2}+d z^{2}\right),
$$

where $q$ is a constant. The Einstein tensor becomes

$$
\mathrm{G}_{\mu \nu}=\rho \xi_{\mu} \xi_{\nu} .
$$

All scalars constructed from the Ricci tensor vanish. We will not consider this case in the following.

\section{Topologically massive gravity theory}

TMG equations were found by Deser, Jackiw and Templeton (DJT) [1]. Recently [3, 4] this theory was extended to the case with a cosmological constant. They are given as follows:

$$
G_{v}^{\mu}+\frac{1}{\mu} C^{\mu}{ }_{v}=\lambda \delta_{v}^{\mu} .
$$

Here $G_{\mu v}$ and $R_{\mu v}$ are the Einstein and Ricci tensors respectively and $C_{v}{ }^{\mu}$ is the Cotton tensor which is given by

$$
C^{\mu}{ }_{\nu}=\eta^{\mu \beta \alpha}\left(R_{v \beta}-\frac{1}{4} R g_{v \beta}\right)_{; \alpha .}
$$

The constants $\mu$ and $\lambda$ are respectively the DJT parameter and the cosmological constant. Solutions of this theory were studied by several authors [10-29].

1. When the spacetime admits a non-null Killing vector field $u^{\mu}$, assuming that the Ricci scalar $R$ is a constant (or the Gaussian curvature, $\mathrm{R}=2 \mathrm{~K}+2 \mathrm{w}^{2}$, of the two spaces orthogonal to the Killing directions is a constant) and using equation (34) we get 


$$
C_{\mu v}=-w\left(3 w^{2}+\frac{R}{2}\right)\left[g_{\mu v}-\frac{3}{\alpha} u_{\mu} u_{v}\right] .
$$

Using the TMG field equations we obtain

$$
\mu=3 w, \quad \lambda=-\frac{R}{6},
$$

which is valid for both spacelike and timelike cases. Metrics (35) with equations (36) and (37) with equation (38) are exact solutions of TMG with parameters satisfying conditions (77).

2. If the spacetime admits a null Killing vector, the Einstein tensor takes the form (61). Using the Killing equation (44) and the Einstein tensor (61) we get

$$
C^{\mu}{ }_{\nu}=(w \rho-\sigma) \xi_{\mu} \xi_{v}, \quad \sigma=\frac{\xi_{1} \rho_{, z}-\xi_{2} \rho_{, r}}{\Delta},
$$

which leads to the following equations:

$$
\begin{aligned}
& \lambda=w^{2}, \\
& (\mu+w) \rho=\sigma=\frac{\xi_{1} \rho_{, z}-\xi_{2} \rho_{, r}}{\Delta}
\end{aligned}
$$

In addition to the Killing equation (64) these equations constitute the field equations to be solved for the DJT theory. With the simplification done in 5.A of the last section we get

$$
\rho=\rho_{0} q^{\frac{\mu+w}{2 w}}, \quad m=-\frac{q, r}{2 w q},
$$

where $\rho_{0}$ is an arbitrary constant. As far as the solutions are concerned we have the following three classes.

(a) We obtain a simple solution of (80) when $\mu=-w$, which leads to $\rho=\rho_{0}$ constant.

Thenusingthesimplificationsdonein5.Bofthelastsection $\quad\left(\xi_{1}=0\right.$ and $\xi_{2}=q=\mathrm{e}^{y}$, $\left.n=m=-\frac{1}{2 w} y_{, r}\right)_{\text {We get all metric functions related to the function } y \text { which satisfies }}$ the differential equation (69). An exact solution and metric of the spacetime is given in (70) of 5.B of the last section.

(b) A more general solution is obtained when $\mu+3 w=0$ where the function $y$ satisfies the equation

$$
-\left(y_{, z z}+y_{, r r}\right)+\frac{1}{2}\left(\left(y_{, r}\right)^{2}+\left(y_{, z}\right)^{2}\right)=\rho_{0} y+\rho_{2},
$$

where $\rho_{2}$ is an integration constant. A solution of this equation is $y=\frac{\rho_{0}}{2} r^{2}, \rho_{2}=$ $-\rho_{0}$. Then the metric becomes

$$
\mathrm{d}^{2}=\mathrm{e}^{\rho_{0} r^{2}} \mathrm{~d} t \mathrm{~d} z+\frac{\rho_{0}^{2} r^{2}}{4 w^{2}}\left[\mathrm{~d} r^{2}+\mathrm{d} z^{2}\right]
$$

Here $\rho_{0} \neq 0$.

(c) The case when $\mu+5 w \neq 0$. The function $y$ satisfies the equation

$$
-\left(y_{, z z}+y_{, r r}\right)+\frac{1}{2}\left(\left(y_{, r}\right)^{2}+\left(y_{, z}\right)^{2}\right)=\frac{\rho_{0}}{\epsilon} \mathrm{e}^{\epsilon y}+\rho_{3},
$$


where $\rho_{3}$ is an integration constant and $\epsilon=\frac{\mu+5 w}{2 w}$. The circularly symmetric metric

$$
s^{2}=\mathrm{e}^{y} \mathrm{~d} t \mathrm{~d} z+\frac{1}{4 w^{2}} \mathrm{e}^{\epsilon y}\left[-\frac{2 \rho_{0}}{\epsilon(\epsilon \quad 1)}+2 \rho_{3} \mathrm{e}^{-\epsilon y}+\rho_{4} \mathrm{e}^{(1-\epsilon) y}\right]\left(\mathrm{d} r^{2}+\mathrm{d} z^{2}\right),
$$

has the form $\mathrm{d}$

where the function $y=y(r)$ satisfies the equation

$$
y_{r}= \pm \mathrm{e}^{\frac{\epsilon}{2} y} \sqrt{-\frac{2 \rho_{0}}{\epsilon(\epsilon-1)}+2 \rho_{3} \mathrm{e}^{-\epsilon y}+\rho_{4} \mathrm{e}^{(1-\epsilon) y}} .
$$

Here $\rho_{4}$ is also an integration constant. All other cases will be considered later.

Remark 4. In the case of the non-null Killing vector field when $w=0$ the Killing vector becomes hypersurface orthogonal and the Cotton tensor vanishes. TMG field equations with hypersurface orthogonal Killing vectors were previously considered. With a hypersurface orthogonal Killing vector Aliev and Nutku showed that TMG allows only a flat solution [40]. Recently Deser and Franklin [41] considered circularly symmetric spacetime in TMG. They showed that such symmetry (existence of the hypersurface orthogonal spacelike Killing vector $\frac{\partial}{\partial \theta}$ ) breaks TMG with a cosmological constant down to vanishing of Cotton and Einstein sectors separately.

\section{A new massive gravity in three dimensions}

Recently a new parity-preserving theory has been introduced by Bergshoeff-HohmTownsend (BHT) [5] in three dimensions that is equivalent to the Pauli-Fierz massive field theory at the linearized level. Originally this theory, known as NMG, did not contain a cosmological term in the action. Adding a cosmological constant $\lambda$ the NMG field equations (CNMG) are given as

$$
2 m_{0}^{2} G_{\mu \nu}+K_{\mu \nu}+\lambda g_{\mu \nu}=0
$$

where

$K_{\mu \nu}=2 \nabla^{2} R_{\mu \nu}-\frac{1}{2}\left(R_{; \mu \nu}+g_{\mu \nu} \nabla^{2} R\right)-8 R_{\mu}^{\rho} R_{\nu \rho}+\frac{9}{2} R R_{\mu \nu}+\left[3 R^{\alpha \beta} R_{\alpha \beta}-\frac{13}{8} R^{2}\right] g_{\mu \nu}$,

where $m_{0}$ is the relative mass parameter and $\nabla^{2}$ is the Laplace-Beltrami operator. Here we used the mass parameter as $m_{0}$ not to confuse with the metric function $m$. Solutions of these equations have been recently studied in $[6,15]$.

1. When the spacetime admits a non-null Killing vector field, assuming that the Ricci scalar $R$ is a constant and using the equation (34) we get $\mathrm{R}=2 \mathrm{~K}+2 \mathrm{w}^{2}$ and 


$$
\begin{aligned}
& \nabla^{2} R_{\mu \nu}=2 w^{2}\left(3 w^{2}+\frac{R}{2}\right)\left[g_{\mu \nu}-\frac{3}{\alpha} u_{\mu} u_{\nu}\right] \\
& R^{\mu \alpha} R_{\nu \alpha}=\frac{1}{\alpha}\left(3 w^{2}+\frac{R}{2}\right)\left(w^{2}-\frac{R}{2}\right) u^{\mu} u_{\nu}+\left(w^{2}+\frac{R}{2}\right)^{2} \delta^{\mu}{ }_{\nu}, \\
& K_{\mu \nu}=-\frac{1}{\alpha}\left(3 w^{2}+\frac{R}{2}\right)\left(20 w^{2}+\frac{R}{2}\right) u_{\mu} u_{\nu}+\left(22 w^{4}+\frac{9}{2} w^{2} R+\frac{R^{2}}{8}\right) g_{\mu \nu}
\end{aligned}
$$

Then using the field equations of the NMG gravity we obtain $\left(3 w^{2}+\frac{R}{2} \neq 0\right)$

$$
m_{0}^{2}=-10 w^{2}-\frac{R}{4}, \quad \lambda=-2 w^{4}-\frac{R^{2}}{8}-4 w^{2} R .
$$

Metrics (35) with equation (36) and (37) with equation (38) are exact solutions of the NMG with parameters satisfying conditions (92). As an example the metric found in [10] as an exact solution of the TMG is also an exact solution of the NMG with parameters satisfying conditions (92).

$$
\begin{aligned}
& \text { If } 3 w^{2}+\frac{R}{2}=0, \text { then we obtain } \\
& \lambda+2 m_{0}^{2} w^{2}+\frac{1}{2} w^{4}=0 .
\end{aligned}
$$

2. When the spacetime admits a null Killing vector field, using equation (44) and the Einstein tensor (61) we obtain $\mathrm{R}=-6 \mathrm{w}^{2}$ and

$$
\begin{aligned}
& \nabla^{2} R_{\mu \nu}=\left(\nabla^{2} \rho+4 w \sigma+6 w^{2} \rho\right) \xi_{\mu} \xi_{\nu}, \\
& R^{\rho}{ }_{\mu} R_{\rho \nu}=-4 w^{2} \rho \xi_{\mu} \xi_{v}+4 w^{4} g_{\mu \nu}, \\
& K_{\mu \nu}=\left[2 \nabla^{2} \rho+8 w \sigma+17 w^{2} \rho\right] \xi_{\mu} \xi_{v}-\frac{1}{2} w^{4} g_{\mu \nu}
\end{aligned}
$$

These equations lead to the following results:

$$
\begin{aligned}
& 2 \nabla^{2} \rho+\left(2 m_{0}^{2}+17 w^{2}\right) \rho+8 w \sigma=0 \\
& \text { (97) } 4 m_{0}^{2} w^{2}-w^{4}+2 \lambda=0 .
\end{aligned}
$$

The full massive gravity field equations reduce to (97) and (98) and equation (44). A circularly symmetric solution of this equation is given by $\rho=\rho_{0} \mathrm{e}^{\mathrm{ky}}$ where $\rho_{0}$ is a constant and $k$ satisfies the quadratic equation $8 k^{2}+24 k+35 / 2-\frac{\lambda}{w^{4}}=0$. The circularly symmetric metric becomes

$$
s^{2}=\mathrm{e}^{2 y} \mathrm{~d} t \mathrm{~d} z+\frac{1}{4 w^{2}}\left[\frac{2 \rho_{0}}{k-1} \mathrm{e}^{k y}+\rho_{1} \mathrm{e}^{y}\right]\left(\mathrm{d} r^{2}+\mathrm{d} z^{2}\right) .
$$

Here $y$ satisfies a similar equation such as (86):

$$
y_{, r}= \pm \sqrt{\frac{2 \rho_{0}}{k-1} \mathrm{e}^{k y}+\rho_{1} \mathrm{e}^{y}}
$$

where $\rho_{1}$ is an integration constant. For each value of $k\left(k_{1}\right.$ and $\left.k_{2}\right)$ we have two different metrics. 
BHT introduced also a more general model [5] which also includes TMG as a special case:

$$
\lambda m_{0}^{2} g_{\mu \nu}+\alpha G_{\mu \nu}+\frac{1}{\mu} C_{\mu \nu}+\frac{\beta}{2 m_{0}^{2}} K_{\mu \nu}=0
$$

where $\lambda, \alpha$ and $\beta$ are the dimensionless parameters. For $\mathrm{m}_{0} \rightarrow \infty$ and for fixed $\mu$ generalized BHT equations reduce to the DJT equations (74). The solutions of these equations are given as follows.

1. Non-null case $\left(3 w^{2}+\frac{R}{2} \neq 0\right)$ :

$$
\begin{aligned}
& -\alpha+\frac{3 w}{\mu}-\frac{\beta}{2 m_{0}^{2}}\left(20 w^{2}+R / 2\right)=0, \\
& \lambda m_{0}^{2}+\alpha w^{2}-\frac{w}{\mu}\left(3 w^{2}+\frac{R}{2}\right)+\frac{\beta}{2 m_{0}^{2}}\left(22 w^{4}+\frac{9}{2} w^{2} R+\frac{R^{2}}{8}\right)=0
\end{aligned}
$$

If $3^{w^{2}+\frac{R}{2}}=0$, then

$$
\lambda m_{0}^{2}+\alpha w^{2}+\frac{\beta}{4 m_{0}^{2}} w^{4}=0 .
$$

2. Null case:

$$
\begin{aligned}
& \frac{\beta}{m_{0}^{2}} \nabla^{2} \rho+\left(\alpha+\frac{w}{\mu}+\frac{17 w^{2} \beta}{2 m_{0} 2}\right) \rho+\left(-\frac{1}{\mu}+\frac{4 \beta w}{m_{0}^{2}}\right) \sigma=0 \\
& \lambda m_{0}^{2}+\alpha w^{2}-\frac{\beta}{4 m_{0}^{2}} w^{4}=0 .
\end{aligned}
$$

In a similar fashion letting $\rho=\rho_{0} \mathrm{e}^{\mathrm{k}} \mathrm{y}$ we get a quadratic equation for $\mathrm{k}$ :

$$
4 w^{2} \frac{\beta}{m_{0}^{2}} k(k+1)+2\left(-\frac{1}{\mu}+\frac{4 \beta w}{m_{0}^{2}}\right) w k+\left(\alpha+\frac{w}{\mu}+\frac{17 w^{2} \beta}{2 m_{0} 2}\right)=0 \text {. }
$$

In order to have the roots exist, the following condition should be satisfied:

$$
\left(-\frac{1}{\mu}+\frac{4 \beta w}{m_{0}^{2}}+2 \frac{\beta^{2}}{m_{0}^{2}}\right)^{2}-4 \frac{\beta}{m_{0}^{2}}\left(\alpha+\frac{w}{\mu}+\frac{17 w^{2} \beta}{2 m_{0} 2}\right) w^{2} \geqslant 0 \text {. }
$$

The metric function $y$ satisfies exactly the same equation (100) but $k$ solves the quadratic equation (107) in this case. The circularly symmetric metric is of the form given in (99), where $y(r)$ satisfies (100).

Both for the null and non-null cases when the scalars of the Killing vector fields are constants then we have a more general result. The following conjecture implies that the corresponding metrics solve all higher derivative gravitational field equations in three dimensions.

Conjecture. Let a three-dimensional spacetime admit a Killing vector field $\mathrm{u}_{\mu}$ (non-null or null) with constant scalars. Let the Gaussian curvature K of the two-dimensional spaces be a constant for the case of non-null vector fields where the corresponding Einstein tensors are 
respectively given in (39) and (61). Then any symmetric covariant second rank tensor constructed from the Ricci tensor by covariant differentiation and by contraction is the linear sum of $\mathrm{u}_{\mu} \mathrm{u}_{v}$ and the metric tensor $\mathrm{g}_{\mu v}$.

\section{Conclusion}

In this work we first studied Killing vector fields in a three-dimensional spacetime geometry. We showed that, independent of the type of the Killing vector fields, the Ricci tensor can be determined in terms of the Killing vector fields and their scalars. Usually the components of this tensor are calculated in terms of the components of the metric tensor in a given coordinate system. In three dimensions, when the geometry admits at least one Killing vector field we do not have to follow such a direction to determine the components of the Ricci tensor. Using this property in each case (when the Killing vector field is timelike, spacelike or null) we first presented the solutions of Einstein field equations with sources. Then by using special cases, when the scalars of the Killing vector fields are constants, we gave solutions of the field equations of the topologically massive gravity and new massive gravity theories. Some of the solutions of the topologically massive gravity field equations which we obtained in this work may already be known. Our basic purpose in this work is to present a new method to solve higher derivative gravity theories rather than finding specific solutions. We conjecture at the end that for the three types of Killing vector fields with constant scalars our method solves all higher derivative theories in three dimensions.

\section{Acknowledgments}

This work is partially supported by the Scientific and Technological Research Council of Turkey (TUBITAK) and Turkish Academy of Sciences (TUBA).

\section{References}

[1] Deser S, Jackiw R and Templeton S 1982 Phys. Rev. Lett. 48975

[2] Deser S, Jackiw R and Templeton S 1982 Ann. Phys., NY 140372 Deser S, Jackiw R and Templeton S 1988 Ann. Phys., NY 185406 (erratum)

[3] Carlip S, Deser S, Waldraon A and Wise D K 2009 Class. Quantum Grav. 26075008 Carlip S, Deser S, Waldraon A and Wise D K 2008 Phys. Lett. B 666272

[4] Song W Ki and Strominger A 2008 J. High Energy Phys. JHEP04(2008)082

[5] Bergshoeff E, Hohm O and Townsend P K 2009 Phys. Rev. Lett. 102201301

[6] Bergshoeff E, Hohm O and Townsend P K 2009 Phys. Rev. D 7924042

[7] Bergshoeff E, Hohm O and Townsend P K 2009 On massive gravitons in 2+1 dimensions Proc. Spanish Relativity Meeting ERE2009 (Bilbao) (arXiv:0912.2944 [hep-th])

[8] Deser S 2009 Phys. Rev. Lett. 103101302

[9] Tekin B and Gull“" u I 2009* Phys. Rev. D 80064033

[10] Gurses M 1994“Class. Quantum Grav. 112585

[11] Clement G 1994 Phys. Rev. 495131

[12] Clement G 1994 Class. Quantum Grav. 11 L115

[13] Moussa K A, Clement G and Leygnac C 2003 Class. Quantum Grav. 20 L277-83

[14] Moussa K A, Clement G, Guennoune H and Leygnac C 2008 Phys. Rev. D 78064065

[15] Clement G 2009 Class. Quantum Grav. 26105015

[16] Clement G 2009 Class. Quantum Grav. 26165002

[17] Palais R S 1979 Commun. Math. Phys. 6919

[18] Fels M E and Torre C G 2002 Class. Quantum Grav. 19641 
[19] Deser S and Tekin B 2003 Class. Quantum Grav. 204877

[20] Chow D D K, Pope C N and Sezgin E 2010 Class. Quantum Grav. 27105001

[21] Anninos D, Li W, Song W and Strominger A 2009 J. High Energy Phys. JHEP03(2009)130

[22] Deser S, Jackiw R and Pi S Y 2005 Cotton blend gravity pp-waves (dedicated to Andrzej Staruszkiewicz, a pioneer in $d=3$ gravity, on his 65th birthday) Acta Phys. Pol. B 36 27-34 (arXiv:gr-qc/0409011)

[23] Ayon-Beato E and Hassaine M 2005 Ann. Phys. 317175

[24] Ayon-Beato E and Hassaine M 2006 Phys. Rev. D 73104001

[25] Chow D D K, Pope C N and Sezgin E 2010 Class. Quantum Grav. 27105002

[26] Gurses M, Karasu A and Sario“ glu $\quad$ O 2005“ Class. Quantum Grav. 22 1527-43

[27] Gurses M and Sario“ glũ O 2005“ Class. Quantum Grav. 224699

[28] Gleiser R J, Gurses M, Karasu A and Sarı” $\quad$ glu $\quad$ O 2006“ Class. Quantum Grav. 232653

[29] Gurses M 2010“Gen. Rel. Grav. 421413

[30] Barrow J D, Shaw D J and Tsagas C G 2006 Class. Quantum Grav. 23 5291-322

[31] Nutku Y 1993 Class. Quantum. Grav. 102657

[32] Vuorio I 1985 Phys. Lett. B 16391

Percacci R, Sodano P and Vuorio I 1987 Ann. Phys., NY 176344

[33] Ayon-Beato E, Giribet G and Hassaine M 2009 J. High Energy Phys. JHEP05(2009)029

[34] Hawking S W and Ellis G F R 1973 The Large Scale Structure of Spacetime (Cambridge: Cambridge University Press)

[35] Carter B 1969 J. Math. Phys. 1070

[36] Banados M, Henneaux M, Teitelboim C and Zanelli J 1993 Phys. Rev. D 481506

[37] Eisenhart L P 1964 Riemannian Geometry 5th edn (Princeton, NJ: Princeton University Press)

[38] Hall G S 1990 J. Math. Phys. 311198

[39] Carot J 2000 Class. Quantum Grav. 172675

[40] Aliev A N and Nutku Y 1996 Class. Quantum Grav. 13 L29-32

[41] Deser S and Franklin J 2010 Class. Quantum Grav. 27107002 